\title{
Candida endophthalmitis: an unusual complication of prolonged intravenous access
}

\author{
$M$ Vose, S Beatty, S J Charles
}

\begin{abstract}
A 16 year old boy awaiting a defunctioning colostomy for Crohn's disease complained of reduced vision in his left eye. Four weeks previously candida had been isolated from his central line used for parenteral feeds. Fundal examination of the left eye revealed a macular abscess with a classic "string of pearls" appearance of multiple vitreous abscesses. This was treated with pars plana vitrectomy and intravitreal antifungal therapy. Microbiological studies confirmed a diagnosis of candida endophthalmitis. (Postgrad Med f 2001;77:119-120)
\end{abstract}

Keywords: candida; endophthalmitis; intravitreal

Candida albicans is the most common causative agent in intraocular fungal infection. Candida endophthalmitis is typically seen in immunocompromised patients, but may also occur in association with intravenous access in the presence of a normal immune system. Examples of intravenous introduction of candida include intravenous drug users ${ }^{1}$; prolonged intravenous/parenteral therapy, ${ }^{2}$ and anaesthetic induction agents.

\section{Case report}

A 16 year old boy was referred with decreased visual acuity and redness of the left eye. Four weeks previously he had candida species isolated from a central line used for parenteral nutrition while awaiting a defunctioning colostomy for Crohn's disease and was started on oral fluconazole $200 \mathrm{mg}$ twice a day. No other predisposing factors (immunodeficiency, corticosteroid treatment, cytotoxic chemotherapy, or prolonged systemic antibiotic treatment ${ }^{4}$ ) were identified.

The visual acuity in the affected eye was reduced to counting fingers. Anterior segment examination was unremarkable but fundos-

Manchester Royal Eye Hospital, Oxford Road, Manchester M13 9WL, UK

$M$ Vose

S Beatty

S J Charles

Correspondence to: Mr Vose

Submitted 27 March 2000 Accepted 11 July 2000

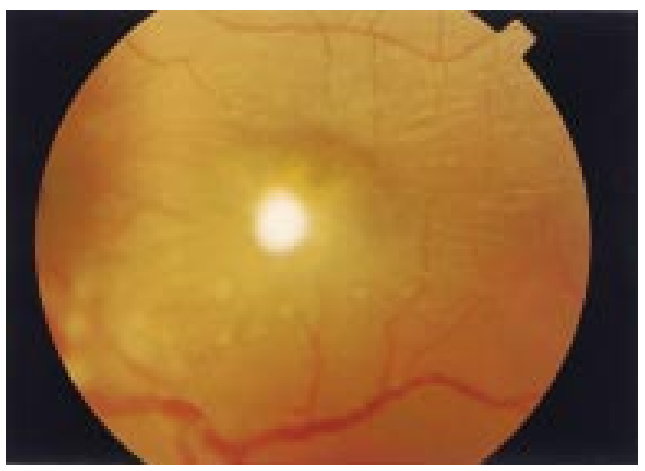

Figure 1 Macular abscess with "string of pearls" inferiorly.

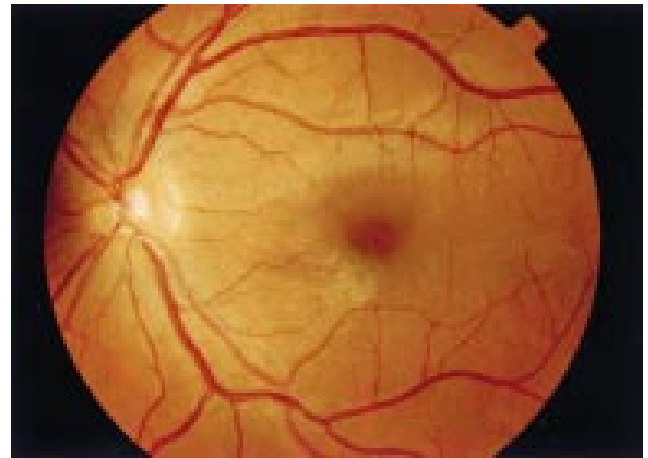

Figure 2 Postoperative appearance six months after vitrectomy.

copy revealed a raised white abscess at the macula with vitritis and microabscesses overlying the inferior arcade (fig 1). Examination of the fellow eye was completely normal. A clinical diagnosis of candida endophthalmitis was made and he was treated with intravitreal amphotericin $(10 \mu \mathrm{g})$ and the dose of fluconazole was increased to $400 \mathrm{mg}$ orally twice daily. One week later, there was no clinical improvement and, therefore, the patient underwent a pars plana vitrectomy with vitreous sampling for microbiological studies and repeat intravitreal injection of amphotericin B $(10 \mu \mathrm{g})$.

Microbiological investigations confirmed the presence of $C$ albicans sensitive to fluconazole. As the patient had already been receiving the appropriate antifungal agent for one month since the contaminated central line had been removed, the dose of fluconazole was increased to $600 \mathrm{mg}$ twice a day.

At six weeks the visual acuity had improved to $6 / 36$ and on review six months later the infection had completely resolved and the patient's visual acuity had improved to 6/9 (fig 2).

\section{Discussion}

Candida species is widespread in the hospital environment and is a commensal organism in the upper respiratory, gastrointestinal, and genitourinary tracts. Consequently, a high index of suspicion of candida endophthalmitis is required in patients with predisposing factors. Blood cultures are frequently negative. ${ }^{5}$ When suspected, prompt ophthalmic referral is advised as intravitreal amphotericin and oral antifungals are highly effective in eradicating the organism and preserving vision. ${ }^{6}$ Fluconazole (a triazole) is the oral antifungal agent of choice. The usual dose is between 200-400 $\mathrm{mg}$ /day in divided doses but up to $1600 \mathrm{mg}$ /day may be used in severe systemic disorders. ${ }^{7}$ Those patients with renal or hepatic impairment, or who are pregnant, need more careful 


\section{Learning points}

1. A high index of suspicion of fungal infection is warranted in patients with the following risk factors:

(A) Intravenous drug use

(B) Immunodeficiency

(C) Prolonged corticosteroid therapy

(D) Cytotoxic chemotherapy

(E) Prolonged systemic antibiotic treatment

(F) Parenteral feeds

2. Early diagnosis of candida endophthalmitis improves visual prognosis

3. Physical/ophthalmic signs may be minimal either because:

(A) Patient's general condition may be poor: intravenous drug use, prolonged intravenous therapy (antibiotics, steroid, cytotoxic, parenteral)

(B) Eye pathology may be very subtle. Patients may present with a slight red eye, photophobia or floaters. Visual acuity may be normal if the macula or the optic nerve are not involved

4. Early lesions may only be evident on fluorescein angiography

5. Anterior segment inflammation (iritis, scleritis, or hypopyon) is a late manifestation of posterior segment infection by candida

6. Transient side effects of empirical antimycotic therapy include: anaemia, thrombocytopenia, increased serum creatinine ${ }^{9}$

7. Urgent specialist ophthalmic opinion is indicated in all cases

8. Oral fluconazole may be started when the diagnosis is suspected ${ }^{10}$

9. Intravitreal antifungal agents are required in those patients with vitreous involvement

10. Early vitrectomy is indicated when the efficacy of therapy (intravenous/intravitreal) is insufficient

increments in their dosage. Vitrectomy is reserved for insufficient clinical improvement, obtaining adequate samples for sensitivity determination and to debulk lesions threatening a retinal detachment. ${ }^{8}$

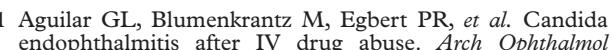
endophthalmitis

2 Servant JB, Dutton GN, Ong-Tone L, et al. Candida endophthalmitis in Glaswegian heroin addicts: report of an epidemic. Trans Ophthalmolol Soc UK 1985;104:297-308.

3 Daily MJ, Dickey JB, Packo KH. Endogenous endophthalmitis after IV anaesthesia with propofol. Arch Ophthalmol 1991;109:1081-4.
4 Edwards JE, Froos RY, Montgomerie JZ, et al. Ocular manifestations of candida septicaemia - review of 76 cases of Candida endophthalmitis. Medicine 1974;53:47-75.

5 Fahey DK, Fenton S, Cahill $M$, et al. Candida endophthalmitis - a diagnostic dilemma. Eye 1999;13:5968.

6 Stern GA, Fetkenhour CL, O'Grady RB. Intravitreal amphotericin B in the treatment of candida endophthalmitis. Arch Ophthalmol 1977;95:89-93.

7 Smiddy WE. Treatment outcomes of endogenous fungal endophthalmitis. Curr Opin Ophthalmol 1998;90:66-70.

8 Towler HMA, Lightman S, Matheson M. Candida endoph8 Towler HMA, Lightman S, Matheson M. C

9 Griffin JR, Pettit TH, Fishman LS, et al. Blood borne Griffin JR, Pettit TH, Fishman LS, et al. Blood borne
candida endophthalmitis; a clinical and pathological study of 21 cases. Arch Ophthalmol 1973;89:450-6.

10 Luttrall JK, Wan W. Treatment of ocular infections with oral fluconazole. Am f Ophthalmol 1995;119:477-81. 